\title{
Vulvovaginal conditions remain difficult and unresolved
}

Vulvovaginal disease is often difficult and problematic. Standard information is often lacking, as may be the knowledge of the individual gynaecologist, who is required to make diagnoses and decide on a management course.

Difficulties associated with vulvar and vaginal conditions may not be as commonplace for the practising gynaecologist as the dilemma of an uncomplicated pregnancy at 40 weeks, or how to perform a challenging caesarean section or how to manage painful and heavy menstruation, but the average gynaecologist must be aware that many patients with vulvar and vaginal conditions are not adequately treated, and that the classification of some conditions often seems incomplete and confusing.

The simplest and yet most complex of these conditions is vulvar thrush, or candidiasis, 'Candida albicans'. Many gynaecologists know that Candida is the genus and Candida albicans a species, one of a multitude of Candida spp., which leads to a difficulty the common treatment of candidiasis, imidazoles, treats primarily the pseudohyphae-forming C. albicans and not its relatives. A patient affected by recurrent thrush will describe how ineffective imidazoles can be. Perhaps this failure of imidazole treatment should be attributed as much to the cyclical or recurring alteration of the vaginal environment, such as $\mathrm{pH}$, as to deficiencies in therapy.

Every student can explain that this changing environment may be caused by loss of the vaginal Lactobacillus spp., which may occur menstrually, with antibiotic use, pregnancy, or hormone treatments. However, this knowledge does not resolve the problem of a person with recurrent thrush who remains affected.

Perhaps it is important to look beyond the Candida genus. Tinea spp. and many other fungi may infect the vulva, as they favour a naturally damp environment. Some are responsive to imidazoles. If feet are a possible source of these fungi, perhaps simple washing of feet last may prevent certain cases of recurring candidiasis that are otherwise inexplicable?

Our understanding of pathogens in the vagina is lacking. Our understanding of the infinitely complex normal vaginal bacterial environment is also deficient: what are the normal organisms of the vagina and vulva?

Mycoplasma spp. have been associated with many conditions, including bacterial vaginosis and pelvic inflammatory disease (PID), which is presumably a condition in which pathogenic organisms ascend from the vagina in association with sexual intercourse and pass into the uterus and adnexa. Mycoplasma and other species have been associated with PID, and yet further reading will show that their behaviour as pathogens is open to considerable debate and that they may also be commensals, found in circumstances where there is no pathology. There are many other organisms, including anaerobic streptococci, for which this is true. And so perhaps it is not the organism itself that is the ratelimiting step in creating a pathological state, but, as with Candida spp., some other factor that stimulates pathogenic behaviour.

This uncertainty of identification of the normal vaginal flora may seem theoretical and academic, but this inability to identify the normal vaginal flora makes it difficult to isolate and identify the abnormal.
A patient with a recurrent or persistent vaginal discharge, with a distressing odour, may respond to metronidazole at first. But then the distressing discharge with no apparent cause, returns, leaving both the patient and gynaecologist frustrated.

The uncertain dilemma of pathogenic/commensal behaviour is well illustrated by bacterial vaginosis, which may or may not be associated with a discharge. Bacterial vaginosis is a condition known to every medical student, having recognised criteria which are easily asked in local and national exams. Yet, bacterial vaginosis may be described as a purely incidental finding on a Pap smear. On questioning, the patient may say that the key component of the discharge is absent, as is any associated local discomfort. This incidental finding of asymptomatic bacterial vaginosis (though it may have obstetric implications, which are also contested) may lead the gynaecologist to prescribe metronidazole - a substance that is unpleasant, may have no benefit, and may even initiate a previously non-existent disturbance of the vaginal flora.

Attempting to find an infective agent has confused the understanding of another condition that has many names and often no recognisable cure - vulvodynia, vulvar vestibulitis, the dysaesthetic vulva - the names can be changed or refined, but the absence of a cure remains. It is difficult to understand how one nomenclature can represent cases without an obvious cure when the majority of cases do. Certain instances may be associated with a recognisable allergic stimulus, a dermatosis or an underlying psychological difficulty, but many are not. They occur in seemingly well individuals in whom an exhaustive search for an allergen or predisposition is fruitless. The condition can remain baffling and frustrating to the patient and to the assisting gynaecologist. Human papilloma virus (HPV) was a favourite cause temporarily, but studies showed identical rates in sufferers and controls, and this association remained nothing more than a case of applying a current focus of research to a condition, without obvious logic or benefit.

Molluscum contagiosum is a condition of the vulva which is virally associated, where small raised lesions exist but do not coalesce to form warty growths. It may be reassuring to know that there are no significant sequelae or that in this case, a simple viral cause may be implicated, but ineffective treatment and recurrence may continue to hamper the patient and physician.

A lack of significant sequelae or association seems not to be the case for Paget's disease of the vulva. The excellent and distinguished American gynaecological oncologist Philip Disaia, co-editor of the current 9th edition of a standard oncology textbook, co-authored a paper in 1989 which identified no patients with vulvar Paget's disease as having an underlying carcinoma during a 10-year follow-up. ${ }^{[1]}$ Studies by himself have since given a different emphasis, and a study published by the MD Anderson Cancer Center in 2017 showed that, of 89 patients who were followed up over 44 years, $46 \%$ had a synchronous or metachronous underlying carcinoma supporting the current recommended management of colonoscopy and cystoscopy on diagnosis, and stringent follow-up. ${ }^{[2]}$ This represents an about-face on a condition fairly recently considered less significant than the more well-known Paget's disease of the breast. 


\section{EDITORIAL}

The classification of intra-epithelial vulvar disease has been a minefield of misunderstanding and correction. The International Society for the Study of Vulvar Disease (ISSVD) has reclassified vulvar disease multiple times over the last 40 years. Terms have come into the classification and disappeared. The current version notes the collaboration of dermatologists, anal specialists, general physicians, pathologists and other interested parties. Perhaps this divergence of skills has contributed to the shifting sea of terms and conditions, and may even have contributed to misunderstanding.

Perhaps, if lichen sclerosus (LS) had kept its original name of lichen sclerosus et atrophicus (it is frequently not atrophic), a recent discovery of a patient who was accidentally on maintenance high-strength topical steroids - likely to cause iatrogenic atrophy and accidental harm - would not have been made. The initial high-strength treatment is better followed by low-strength maintenance treatment, a protocol known to many. Sometimes, even inadvertently, there may be a certain logic to a nomenclature that has been forgotten.
The vulva and vagina may seem the source of a simple understanding of simple conditions, but scrutiny shows that that understanding is changing constantly, and that many fundamental deficiencies in that understanding still exist.

\section{William Edridge \\ Editor \\ william.edridge@gmail.com}

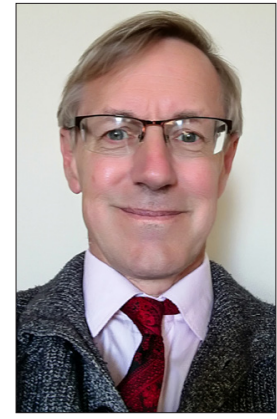

S Afr J Obstet Gynaecol 2018;24(1):2-3. DOI:10.7196/SAJOG.2018.v24i1.1362

1. Bergen S, DiSaia PJ, Liao SY, Berman ML. Conservative management of extramammary Paget's disease of the vulva. Gynecol Oncol 1989;33(2):151-156. https://doi.org/10.1016/00908258(89)90541-6

2. Onaiwu CO, Salcedo MP, Pessini SA, et al. Paget's disease of the vulva: A review of 89 cases. Gynecol Oncol Rep 2017;19:46-49. https://doi.org/10.1016/j.gore.2016.12.010 